\title{
Prilocaine hydrochloride $2 \%$ hyperbaric solution for intrathecal injection: a clinical review
}

\author{
Alberto Manassero' \\ Andrea Fanelli² \\ 'Department of Emergency and \\ Critical Care, Anesthesia and Intensive \\ Care Unit, S. Croce e Carle Hospital, \\ Cuneo, ${ }^{2}$ Department of Medical \\ and Surgical Sciences, Anesthesia \\ and Intensive Care Unit, Policlinico \\ S. Orsola-Malpighi, Bologna, Italy
}

This article was published in the following Dove Press journal:

Local and Regional Anesthesia

3I March 2017

Number of times this article has been viewed

Correspondence: Alberto Manassero Corso IV Novembre 12, Cuneo I2100, $\mathrm{CN}$, Italy

Tel +390I7 I64 2025

$\mathrm{Fax}+390171642010$

Email manassero.al@ospedale.cuneo.it
Abstract: Prilocaine is a local anesthetic characterized by intermediate potency and duration and fast onset of action. As hyperbaric formulation of 5\% solution, it was introduced and has been successfully used for spinal anesthesia since 1960. A new formulation of $2 \%$ plain and hyperbaric solution is currently available in Europe. Because of its lower incidence of transient neurological symptoms, prilocaine is suggested as substitute to lidocaine and mepivacaine in spinal anesthesia for ambulatory surgery, as well as a suitable alternative to low doses of long-acting local anesthetics. The National Library of Medicine database, the Excerpta Medica database, the Cochrane Database of Systematic Reviews, and the Cochrane Central Register of Controlled Trials database, were searched for the period 1970 to September 2016, with the aim to identify studies evaluating the intrathecal use of $2 \%$ prilocaine. A total of 13 randomized clinical trials (RCTs), 1 observational study, 2 dose finding, and 4 systematic reviews has been used for this review. The studies evaluated showed that $2 \%$ hyperbaric prilocaine due to a favorable anesthetic and safety profile is an alternative drug to lidocaine and mepivacaine for spinal anesthesia of intermediate or short duration. In comparison with plain solutions, hyperbaricity remarkably accelerates the onset and offset times of intrathecal $2 \%$ prilocaine. Literature suggests a dose ranging between 40 and $60 \mathrm{mg}$ of prilocaine for lower extremities and lower abdominal procedures lasting up to $90 \mathrm{~min}$, whereas a dose ranging from 10 to $30 \mathrm{mg}$ is appropriate for perineal surgery. Readiness for discharge occurs in $\sim 4 \mathrm{~h}$ from spinal administration.

Keywords: short acting local anesthetic, transient neurologic symptoms, postoperative urinary retention, spinal anesthesia, day surgery

\section{Introduction}

In the last decade, as the trend toward ambulatory surgery continues, interest in available drugs for outpatient spinal anesthesia has increased accordingly. An ideal outpatient spinal anesthetic would provide rapid sensory and motor block, predictable regression and a low incidence of side effects. ${ }^{1}$ For many years this profile has been fulfilled by lidocaine but, in 1993, "transient neurologic symptoms" (TNS) were described as adverse events in patients after a single spinal injection of lidocaine. ${ }^{2,3}$ TNS are known to occur even with other local anesthetics, such as mepivacaine (AstraZeneca, Cambridge, UK) and procaine (Hospira, Inc., Lake Forest, IL, USA). ${ }^{4-6}$ Nevertheless, the highest risk of TNS is related to spinal lidocaine, and therefore its use for spinal anesthesia has been questioned.

Small doses of long-acting local anesthetics such as bupivacaine (AstraZeneca), levobupivacaine (AbbVie Inc., North Chicago, IL, USA), and ropivacaine (AstraZeneca) have been used for spinal anesthesia in ambulatory surgery. With the use of large doses 
of long-acting local anesthetics, delay of discharge emerged as a growing problem, although small doses demonstrated a wide variability in block duration and failure rate..$^{7-8}$

Due to its intermediate duration of action and the lower incidence of TNS, prilocaine (Sintetica SA, Mendrisio, Switzerland) has been proposed as a valuable alternative to lidocaine as well as to small doses of long-acting local anesthetics for short procedures performed under spinal anesthesia.

The purpose of this review is to summarize the available data on the use of prilocaine for spinal anesthesia, focusing on $2 \%$ hyperbaric solutions, to highlight the safety and the effectiveness of its use for ambulatory and short time surgeries.

\section{Methods}

The National Library of Medicine database (MEDLINE), the Excerpta Medica database (EMBASE), the Cochrane Database of Systematic Reviews (CINAHL), and the Cochrane Central Register of Controlled Trials database (CENTRAL) were searched for the time period 1970 to September 2016, with the aim to identify studies evaluating the intrathecal use of prilocaine, rather than studies that compared its intrathecal administration to another anesthetic, in terms of safety, efficacy, and readiness for discharge after ambulatory surgery. Search strategies included the terms "prilocaine" with "spinal anesthesia" and "ambulatory surgery." References of all retrieved articles were manually searched to identify any other study not found in the electronic search.

The quality of the randomized controlled trials (RCTs) included in this review has been reported using the Jadad Scale. ${ }^{9}$ Studies were excluded if they were conference proceedings not followed up by full publication. Only Englishlanguage articles and articles with sufficiently detailed abstract translated into English were included in the review. Randomized trials with a Jadad Scale lower than 3 were excluded.

\section{Results}

A total of 13 RCTs, 1 observational study, 2 dose finding, and 4 systematic reviews has been finally used for this review. The main clinical characteristics of the RCTs using $2 \%$ prilocaine and their Jadad Scale are detailed in Table 1.

\section{Prilocaine}

Prilocaine is an amide-type local anesthetic characterized by intermediate potency and duration and fast onset of action. Unlike lidocaine, which is a tertiary amine, prilocaine is a secondary amine, which has relevant advantages in terms of toxicity. Prilocaine has the highest clearance of all amino-amide local anesthetics, more than twice the clearance of lidocaine.

Together with its larger distribution volume, this feature is responsible for the considerably lower prilocaine plasma concentration compared to lidocaine and mepivacaine after regional anesthesia. ${ }^{10}$ As a consequence, prilocaine reaches toxic blood concentrations very rarely, and the recommended maximum dose is about twice the maximum dose of lidocaine.

The amide-linked local anesthetics are degraded by the hepatic endoplasmic reticulum. In the liver, prilocaine is primarily metabolized by amide hydrolysis to $\sigma$-toluidine and $N$-propylalanine. $\sigma$-Toluidine is subsequently hydroxylated to 2-amino-3-hydroxytoluene and 2-amino-5-hydroxytoluene, metabolites responsible for the occurrence of methemoglobinemia. ${ }^{11} \mathrm{~A}$ high dose of prilocaine (>600 mg) is needed to cause a clinically apparent methemoglobinemia in the healthy adult. ${ }^{12}$ The low doses of hyperbaric prilocaine used in spinal anesthesia do not produce a sufficient amount of $\sigma$-toluidine thereby avoiding additional risk for the patient.

Prilocaine hydrochloride was synthesized in 1953 and submitted to extensive pharmacological and toxicological investigations since the 1960s.

One of the first publications reporting about the intrathecal use of prilocaine appeared in $1965 .{ }^{13}$ The drug (75 mg, 5\% concentration) was administered to 106 patients to achieve spinal anesthesia for transurethral prostate resection. The quality of the block was adequate, safe, and satisfactory.

Prilocaine was never approved for intrathecal administration in the USA, whereas it was used as a standard drug for spinal anesthesia in UK until 1978 and in France until 1998. Despite its favorable anesthetic profile, these products, Citanest 5\% Heavy (AstraZeneca) and Citanest Rachianesthésie (Laboratoires Dentoria, Gentilly, France), were withdrawn from the market for commercial reasons and because of stability problems related to the production procedures. ${ }^{14,15}$

Since 2005, in Germany by AstraZeneca and in Switzerland by Sintetica SA, 2\% prilocaine hydrochloride has been developed as plain and hyperbaric solution, respectively.

\section{2\% Hyperbaric prilocaine}

The solution of $2 \%$ hyperbaric prilocaine developed in Switzerland by Sintetica, contains $6 \%$ glucose and has a density ranging from 1.024 to $1.027 \mathrm{~g} / \mathrm{g}$ at $20^{\circ} \mathrm{C}$, corresponding to a mean density value of 1.021 at $37^{\circ} \mathrm{C}$, higher than the cerebrospinal fluid density at $37^{\circ} \mathrm{C}$. 
It is well known that baricity of the injected drugs mainly affects their spinal spread. ${ }^{16,17}$ These solutions lead to a faster spread to a higher median dermatomal level with less variation in maximum sensory and motor block in comparison with isobaric solutions. ${ }^{18,19} \mathrm{~A}$ more predictable and reliable block follows after hyperbaric than plain solutions. ${ }^{20}$

The distribution of hyperbaric spinal anesthesia also influences the duration of the block. With the same dose of hyperbaric bupivacaine, the spinal block lasted longer in patients with a restricted block. ${ }^{21}$ For this property, several local anesthetics have been formulated as hyperbaric solutions for intrathecal administration. ${ }^{17}$

To investigate the advantages of the hyperbaric formulation versus plain prilocaine, in 2010 Camponovo et al published a randomized, noninferiority study. ${ }^{22}$ The authors compared the efficacy in inducing sensory block to T10 of two different intrathecal doses of $2 \%$ hyperbaric prilocaine (60 and $40 \mathrm{mg}$ ) and one dose of $2 \%$ plain prilocaine (60 mg) in outpatients undergoing elective short-duration surgery (<60 min). Both 60 and $40 \mathrm{mg}$ of $2 \%$ hyperbaric prilocaine induced a T10 level of sensory block with significantly shorter onset times than $60 \mathrm{mg}$ of $2 \%$ plain prilocaine. On recovery from spinal anesthesia, the effects of the hyperbaric solutions ceased more rapidly than the plain solution. In particular, 40 $\mathrm{mg}$ of $2 \%$ hyperbaric prilocaine allowed a complete recovery from the motor block (i.e., time to unassisted ambulation) in $90 \mathrm{~min}$ in comparison with 121 and $160 \mathrm{~min}$ for Group Hyperbaric 60 and Plain 60. In conclusion, 2\% hyperbaric prilocaine remarkably improves the well-known features of the plain solution and shows good suitability for short-duration surgery. Motor and sensory blocks are established faster; the anesthetic is fixed earlier, and patients recover faster after hyperbaric than after spinal plain prilocaine.

Hyperbaric prilocaine 2\% was first compared by Ratsch et al with $0.5 \%$ hyperbaric bupivacaine. ${ }^{23}$ Eighty-eight patients scheduled for lower limb surgery lasting a maximum of 45 min under spinal anesthesia were randomly allocated to receive either $15 \mathrm{mg}$ of $0.5 \%$ hyperbaric bupivacaine or $60 \mathrm{mg} \mathrm{2 \%}$ hyperbaric prilocaine. Both groups were comparable in reaching the required analgesic level of T12, as well as in block intensity and onset times of maximum sensory block. A T12 analgesic level was maintained for $60 \mathrm{~min}$ with prilocaine versus 120 min with bupivacaine, whereas regression of the motor block took 135 versus $210 \mathrm{~min}$ and time for spontaneous micturition was 306 versus $405 \mathrm{~min}$ for prilocaine and bupivacaine, respectively. The two study drugs achieved the equivalent quality of sensory/motor blocks, allowing adequate surgical anesthesia for at least
$1 \mathrm{~h}$, as well as the comparable occurrence of undesired side effects. Nevertheless, $2 \%$ hyperbaric prilocaine was superior to $0.5 \%$ hyperbaric bupivacaine regarding faster offset, faster time to first spontaneous voiding, faster recovery-room and home discharges.

The use of hyperbaric solutions can further allow restricting the block mostly to the operative side. ${ }^{24}$ Unilateral spinal anesthesia allows minimizing the extent of sympathetic blockade, resulting in minimal impairment of cardiovascular homeostasis and thus reducing the incidence of clinically relevant hypotension to $5 \%-7 \% .{ }^{25}$ A more profound motor block to the operative side was also enhanced, increasing patient satisfaction and resulting in a significant acceleration of patient discharge, making unilateral spinal anesthesia an interesting option for outpatient surgery.

For this purpose, Manassero et al compared the anesthetic profile of unilateral and conventional bilateral spinal anesthesia with the same dose $(50 \mathrm{mg})$ of hyperbaric $2 \%$ prilocaine in inguinal herniorrhaphy. ${ }^{26}$ In the unilateral group, spinal anesthesia was performed on lateral decubitus which was maintained for $10 \mathrm{~min}$. With this short time, only $12.5 \%$ of the patients achieved a restrict unilateral spinal block (sensory block below $\mathrm{S} 1$ in the nonoperated limb) 20 min after spinal anesthesia. Nevertheless, time to voiding was faster in the unilateral $(220 \pm 47 \mathrm{~min})$ than in a conventional group (249 $\pm 51 \mathrm{~min})$, demonstrating that attempting unilateral spinal block may improve the time to first voiding and so the time to home discharge. No episodes of urinary retention occurred in either group. The study confirmed prilocaine as an effective spinal anesthetic for day-case surgery showed a dose of $50 \mathrm{mg}$ hyperbaric adequate for inguinal repair lasting up $60 \mathrm{~min}$.

\section{Perianal surgery}

A dose finding study was performed by Gebhardt who tested three dosages of $2 \%$ hyperbaric prilocaine in perianal outpatient surgery. The authors injected 10,20 , or $30 \mathrm{mg}$ in sitting position; then patients were brought into lithotomy position after $10 \mathrm{~min}$. The results suggested $10 \mathrm{mg}$ as the recommended dose in procedures lasting no longer than $40 \mathrm{~min}$, because of sufficient analgesia, preserved motor function of the lower extremities, shorter voiding (173 $\pm 31 \mathrm{~min})$ and discharge time (199 $\pm 39 \mathrm{~min})$. None of the patients suffered from urinary retention. ${ }^{27}$

Accordingly, $10 \mathrm{mg}$ of $2 \%$ hyperbaric prilocaine was then compared by Gebhardt with $20 \mathrm{mg}$ of $4 \%$ hyperbaric mepivacaine in perianal outpatient surgery. The recovery profile from spinal anesthesia with prilocaine $10 \mathrm{mg}$ was confirmed: prilocaine led to shorter time to first spontaneous micturition 
Table I Main results of randomized controlled trials published about prilocaine $2 \%$

\begin{tabular}{|c|c|c|c|c|c|c|c|}
\hline Author & $\begin{array}{l}\text { Jadad } \\
\text { Scale }\end{array}$ & Drugs & Additives & Pts & Setting & $\begin{array}{l}\text { Sensory block } \\
\text { onset (min) }\end{array}$ & $\begin{array}{l}\text { Motor block } \\
\text { onset (min) }\end{array}$ \\
\hline \multirow[t]{2}{*}{$\begin{array}{l}\text { Ambrosoli } \\
\text { et } \mathrm{al}^{31}\end{array}$} & 3 & $40 \mathrm{mg} 2 \% \mathrm{H}$ prilocaine & None & 50 & $\begin{array}{l}\text { Arthroscopic knee } \\
\text { surgery }\end{array}$ & $\begin{array}{l}6.0(5.0-10.0) \text { (in the } \\
\text { femoral nerve } \\
\text { distribution) }\end{array}$ & $\begin{array}{l}6.5(4.0-10.0) \text { (in the } \\
\text { femoral nerve } \\
\text { distribution) }\end{array}$ \\
\hline & & $\begin{array}{l}\text { US-guided femoral-sciatic } \\
\text { nerve block with } 2 \% \\
\text { mepivacaine } 25 \mathrm{~mL}\end{array}$ & None & 50 & & NR & NR \\
\hline \multirow[t]{2}{*}{$\begin{array}{l}\text { Aguirre } \\
\text { et }\left.a\right|^{32}\end{array}$} & 5 & $60 \mathrm{mg} 2 \% \mathrm{H}$ prilocaine & None & 70 & $\begin{array}{l}\text { Arthroscopic knee } \\
\text { surgery }\end{array}$ & $\begin{array}{l}4.2 \pm I \text { (TI0 } \\
\text { dermatome) }\end{array}$ & NR \\
\hline & & $12 \mathrm{mg} 0.4 \% \mathrm{P}$ ropivacaine & None & 70 & & $\begin{array}{l}5.2 \pm I \text { (TI0 } \\
\text { dermatome) }\end{array}$ & NR \\
\hline \multirow[t]{2}{*}{$\begin{array}{l}\text { Manassero } \\
\text { et } \mathrm{al}^{26}\end{array}$} & 3 & $\begin{array}{l}50 \mathrm{mg} 2 \% \mathrm{H} \text { prilocaine } \\
\text { lateral position }\end{array}$ & None & 40 & $\begin{array}{l}\text { Inguinal } \\
\text { herniorrhaphy }\end{array}$ & NR & $\begin{array}{l}\text { At } 10 \text { min } 96 \% * \text { pts } \\
\text { Bromage }=3 \text { in the } \\
\text { operated limb }\end{array}$ \\
\hline & & $\begin{array}{l}50 \mathrm{mg} 2 \% \mathrm{H} \text { prilocaine } \\
\text { sitting position }\end{array}$ & None & 40 & & NR & $\begin{array}{l}\text { At } 10 \text { min } 58 \% \text { pts } \\
\text { Bromage }=3 \text { in the } \\
\text { operated limb }\end{array}$ \\
\hline \multirow[t]{2}{*}{$\begin{array}{l}\text { Kaban } \\
\text { et } \mathrm{al}^{29}\end{array}$} & 3 & $30 \mathrm{mg} 2 \% \mathrm{H}$ prilocaine & $\begin{array}{l}20 \mu g \\
\text { fentanyl }\end{array}$ & 25 & Perianal surgery & $\begin{array}{l}4.6 \pm 1.3^{*}(\mathrm{LI} \\
\text { dermatome) }\end{array}$ & $N R$ \\
\hline & & $\begin{array}{l}7.5 \mathrm{mg} 0.5 \% \mathrm{H} \\
\text { bupivacaine }\end{array}$ & $\begin{array}{l}20 \mu g \\
\text { fentanyl }\end{array}$ & 25 & & $\begin{array}{l}5.9 \pm 1.9(\mathrm{LI} \\
\text { dermatome })\end{array}$ & NR \\
\hline Gebhardt & 4 & $10 \mathrm{mg} 2 \% \mathrm{H}$ prilocaine & None & 80 & Perianal surgery & NR & NR \\
\hline et $\mathrm{al}^{28}$ & & $20 \mathrm{mg} \mathrm{4 \%} \mathrm{H}$ mepivacaine & None & 80 & & NR & NR \\
\hline
\end{tabular}

\begin{tabular}{|c|c|c|c|c|c|c|c|}
\hline \multirow[t]{2}{*}{$\begin{array}{l}\text { Akcaboy } \\
\text { et } \mathrm{a}^{37}\end{array}$} & 5 & $50 \mathrm{mg} 2 \% \mathrm{H}$ prilocaine & $\begin{array}{l}25 \mu \mathrm{g} \\
\text { fentanyl }\end{array}$ & 30 & $\begin{array}{l}\text { Transurethral } \\
\text { resection of } \\
\text { prostate surgery }\end{array}$ & $\begin{array}{l}7.1 \pm 1.9 \text { higher } \\
\text { dermatome }\end{array}$ & $\begin{array}{l}\text { Bromage } 2(\mathrm{I}-3)^{*} \text { (at the } \\
\text { time of reaching highest } \\
\text { sensory block) }\end{array}$ \\
\hline & & $4 \mathrm{mg} 0.5 \% \mathrm{H}$ bupivacaine & $\begin{array}{l}25 \mu \mathrm{g} \\
\text { fentanyl }\end{array}$ & 30 & in geriatric patients & $\begin{array}{l}7.6 \pm 1.3 \text { higher } \\
\text { dermatome }\end{array}$ & $\begin{array}{l}\text { Bromage I (0-3) (at the } \\
\text { time of reaching highest } \\
\text { sensory block) }\end{array}$ \\
\hline \multirow[t]{2}{*}{ Black et $\mathrm{al}^{36}$} & 5 & $20 \mathrm{mg} 2 \% \mathrm{P}$ prilocaine & $\begin{array}{l}20 \mu g \\
\text { fentanyl }\end{array}$ & 25 & $\begin{array}{l}\text { Arthroscopic knee } \\
\text { surgery }\end{array}$ & $\begin{array}{l}\text { I I.3 }(2.5-55) * \text { higher } \\
\text { dermatome }\end{array}$ & NR \\
\hline & & $\begin{array}{l}7.5 \mathrm{mg} 0.5 \% \mathrm{P} \\
\text { bupivacaine }\end{array}$ & $\begin{array}{l}20 \mu g \\
\text { fentanyl }\end{array}$ & 25 & & $\begin{array}{l}20.0(7.5-60) \text { higher } \\
\text { dermatome }\end{array}$ & NR \\
\hline \multirow[t]{3}{*}{$\begin{array}{l}\text { Camponovo } \\
\text { et al }\left.\right|^{22}\end{array}$} & 4 & $40 \mathrm{mg} 2 \% \mathrm{H}$ prilocaine & None & 30 & $\begin{array}{l}\text { Surgical procedures } \\
\text { lasting }<60 \mathrm{~min}\end{array}$ & $\begin{array}{l}9 \pm 5^{a} \text { (TIO } \\
\text { dermatome) }\end{array}$ & $8 \pm 5^{a}($ Bromage $\geq 2)$ \\
\hline & & $60 \mathrm{mg} 2 \% \mathrm{H}$ prilocaine & None & 30 & & $7 \pm 4$ a $(\mathrm{TI} 0$ dermatome $)$ & $8 \pm 3^{a}($ Bromage $\geq 2)$ \\
\hline & & $60 \mathrm{mg} 2 \% \mathrm{P}$ prilocaine & None & 30 & & $\begin{array}{l}14 \pm 7 \text { ( } \mathrm{T} 10 \\
\text { dermatome) }\end{array}$ & $12 \pm 5$ (Bromage $\geq 2)$ \\
\hline \multirow[t]{2}{*}{$\begin{array}{l}\text { Hendriks } \\
\text { et a }\left.\right|^{35}\end{array}$} & 5 & $50 \mathrm{mg} 2 \% \mathrm{P}$ prilocaine & None & 36 & $\begin{array}{l}\text { Arthroscopic knee } \\
\text { surgery }\end{array}$ & $\begin{array}{l}2(2-10)(\mathrm{LI} \\
\text { dermatome) }\end{array}$ & $5(2-15)($ Bromage $=2)$ \\
\hline & & $50 \mathrm{mg} 2 \%$ P Articaine & None & 36 & & $\begin{array}{l}2(2-15)(\mathrm{LI} \\
\text { dermatome) }\end{array}$ & $5(2-15)($ Bromage $=2)$ \\
\hline \multirow[t]{2}{*}{$\begin{array}{l}\text { Rätsch } \\
\text { et } \mathrm{a}^{23}\end{array}$} & 5 & $60 \mathrm{mg} 2 \% \mathrm{H}$ prilocaine & None & 44 & $\begin{array}{l}\text { Lower extremity } \\
\text { procedures lasting }\end{array}$ & $\begin{array}{l}5 \pm 3 \text { (TI2 } \\
\text { dermatome) }\end{array}$ & $10 \pm 10($ Bromage $=3)$ \\
\hline & & $\begin{array}{l}\mathrm{I} 5 \mathrm{mg} 0.5 \% \mathrm{H} \\
\text { bupivacaine }\end{array}$ & None & 44 & up to $45 \mathrm{~min}$ & $\begin{array}{l}4 \pm 8(\mathrm{~T} 12 \\
\text { dermatome) }\end{array}$ & $10 \pm 10($ Bromage $=3)$ \\
\hline
\end{tabular}




\begin{tabular}{|c|c|c|c|c|c|c|}
\hline $\begin{array}{l}\text { Sensory block } \\
\text { resolution ( } \mathrm{min})\end{array}$ & $\begin{array}{l}\text { Motor block } \\
\text { resolution (min) }\end{array}$ & $\begin{array}{l}\text { Time to } \\
\text { micturition (min) }\end{array}$ & POUR & $\begin{array}{l}\text { Fluid } \\
\text { management }\end{array}$ & TNS & Main results \\
\hline NR & $\begin{array}{l}285(240-330)^{*} \\
\text { (unassisted } \\
\text { ambulation) }\end{array}$ & $225(220-300)$ & 0 & $\begin{array}{l}500 \mathrm{~mL} \\
\text { crystalloids } \\
\text { before SA; no }\end{array}$ & 0 & $\begin{array}{l}\text { Discharge home was faster after } \\
\text { intrathecal anesthesia with } 40 \mathrm{mg} \\
\text { hyperbaric prilocaine than after femoral- }\end{array}$ \\
\hline NR & $\begin{array}{l}328(280-362) \\
\text { (unassisted } \\
\text { ambulation) }\end{array}$ & $220(135-290)$ & 0 & $\begin{array}{l}\text { intra-operative } \\
\text { fluids unless } \\
\text { hypotension }\end{array}$ & l & $\begin{array}{l}\text { sciatic nerve blockade following day-case } \\
\text { knee arthroscopy }\end{array}$ \\
\hline $\begin{array}{l}120(120-180) \\
(\mathrm{T} 12 \text { regression })\end{array}$ & $\begin{array}{l}180(169-240)^{*} \\
\text { (Bromage=I) }\end{array}$ & $250(231-300)$ & 0 & $\begin{array}{l}4 \mathrm{~mL} / \mathrm{kg} / \mathrm{h} \text { of } \\
\text { crystalloids }\end{array}$ & 0 & $\begin{array}{l}\text { The recovery of motor block was faster } \\
\text { after } 2 \% \text { prilocaine compared with } 0.4 \%\end{array}$ \\
\hline $\begin{array}{l}120(70-180) \\
(T 12 \text { regression })\end{array}$ & $\begin{array}{l}240(180-300) \\
(\text { Bromage }=1)\end{array}$ & $270(235-320)$ & 0 & $\begin{array}{l}\text { throughout the } \\
\text { procedure }\end{array}$ & 0 & $\begin{array}{l}\text { plain ropivacaine. Discharge time was } \\
\text { similar between the two groups }\end{array}$ \\
\hline $\begin{array}{l}156 \pm 30 \\
\text { ( } 22 \text { regression) in } \\
\text { the operated limb }\end{array}$ & $\begin{array}{l}\text { II } \pm 26 \\
\text { (Bromage }=0) \\
\text { in the operated limb }\end{array}$ & $220 \pm 47 *$ & 0 & $\begin{array}{l}7 \mathrm{~mL} / \mathrm{kg} \\
\text { crystalloids } \\
\text { before SA; }\end{array}$ & 0 & $\begin{array}{l}\text { In day-case inguinal herniorrhaphy, } \\
\text { attempting unilateral spinal anesthesia } \\
\text { with } 50 \mathrm{mg} \text { hyperbaric } 2 \% \text { prilocaine }\end{array}$ \\
\hline $\begin{array}{l}158 \pm 26 \\
\text { ( } 22 \text { regression) in } \\
\text { the operated limb }\end{array}$ & $\begin{array}{l}108 \pm 24 \\
(\text { Bromage }=0) \\
\text { in the operated limb }\end{array}$ & $249 \pm 51$ & 0 & $\begin{array}{l}4 \mathrm{~mL} / \mathrm{kg} / \mathrm{h} \text { until } \\
\text { spontaneous } \\
\text { micturition }\end{array}$ & 0 & produced faster time to voiding \\
\hline $\begin{array}{l}\text { I33 } \pm\left. 4\right|^{*} \\
\text { (S3 regression) }\end{array}$ & $\begin{array}{l}136 \pm 53^{*} \text { (unassisted } \\
\text { ambulation) }\end{array}$ & $152 \pm 104$ & 1 & $\begin{array}{l}7 \mathrm{~mL} / \mathrm{kg} \\
\text { crystalloids }\end{array}$ & 0 & $\begin{array}{l}\text { Prilocaine } 30 \mathrm{mg}+20 \mu \mathrm{g} \text { fentanyl provides } \\
\text { faster sensory block resolution and }\end{array}$ \\
\hline $\begin{array}{l}200 \pm 64 \\
(S 3 \text { regression })\end{array}$ & $\begin{array}{l}\text { I72 } \pm 82 \text { (unassisted } \\
\text { ambulation) }\end{array}$ & $172 \pm 130$ & 1 & $\begin{array}{l}\text { before SA; no } \\
\text { intra-operative } \\
\text { fluids unless } \\
\text { hypotension }\end{array}$ & 0 & $\begin{array}{l}\text { home readiness compared to } 7.5 \mathrm{mg} \\
\text { bupivacaine }+20 \mu \mathrm{g} \text { fentanyl }\end{array}$ \\
\hline NR & $168(98-252)$ & I 78 ( $\mid 10-254)$ & 0 & A maximum & 0 & Both, hyperbaric mepivacaine and \\
\hline NR & $175(100-300)$ & $195(130-305)$ & 0 & $\begin{array}{l}\text { of } 500 \mathrm{ml} \\
\text { crystalloids }\end{array}$ & 6 & $\begin{array}{l}\text { prilocaine can be used at dosage of } 0.5 \\
\mathrm{~mL} \text { each for spinal anesthesia in perianal } \\
\text { outpatient surgery. TNS was lower with } \\
\text { prilocaine. }\end{array}$ \\
\hline NR & $\begin{array}{l}158 \pm 12 \\
(\text { Bromage }=0)\end{array}$ & $\begin{array}{l}\text { Transurethral } \\
\text { catheter }\end{array}$ & & $\begin{array}{l}8 \mathrm{~mL} / \mathrm{kg} / \mathrm{h} \text { of } \\
\text { crystalloids } \\
\text { throughout the }\end{array}$ & NR & $\begin{array}{l}\text { Intrathecal } 4 \mathrm{mg} \text { bupivacaine }+25 \mu \mathrm{g} \\
\text { fentanyl provided adequate spinal } \\
\text { anesthesia with shorter block duration }\end{array}$ \\
\hline NR & $\begin{array}{l}|10 \pm| 4^{*} \\
(\text { Bromage }=0)\end{array}$ & $\begin{array}{l}\text { Transurethral } \\
\text { catheter }\end{array}$ & & procedure & NR & $\begin{array}{l}\text { than intrathecal } 50 \mathrm{mg} \text { prilocaine }+25 \\
\mu \mathrm{g} \text { fentanyl for day case transurethral } \\
\text { resection of prostate surgery in geriatric } \\
\text { patients }\end{array}$ \\
\hline $\begin{array}{l}97(90-115)^{*} \\
\text { (L4 regression) }\end{array}$ & $\begin{array}{l}75 \%{ }^{\mathrm{a}} \text { pts Bromage }=0 \\
\text { at I } \mathrm{h}\end{array}$ & $\begin{array}{l}205 \\
(185-220)^{*}\end{array}$ & 0 & NR & 0 & $\begin{array}{l}\text { Prilocaine showed a faster attainment and } \\
\text { resolution of block, together with greater }\end{array}$ \\
\hline $\begin{array}{l}280 \text { (207-not } \\
\text { computable) } \\
\text { (L4 regression) }\end{array}$ & $\begin{array}{l}\text { None pts } \\
\text { Bromage }=0 \text { at I h }\end{array}$ & $275(250-300)$ & 0 & & 0 & hemodynamic stability \\
\hline $\begin{array}{l}110 \pm 35^{*} \\
\text { (complete } \\
\text { regression) }\end{array}$ & $\begin{array}{l}92 \pm 36^{*} \\
(\text { Bromage }=0)\end{array}$ & $195^{a}(60)$ & 0 & $\begin{array}{l}7 \mathrm{~mL} / \mathrm{kg} \\
\text { crystalloids } \\
\text { before } \mathrm{SA}\end{array}$ & 0 & $\begin{array}{l}2 \% \text { hyperbaric prilocaine showed faster } \\
\text { times to motor block onset and shorter } \\
\text { duration of surgical block }\end{array}$ \\
\hline $\begin{array}{l}132 \pm 34^{*} \\
\text { (complete } \\
\text { regression) }\end{array}$ & $\begin{array}{l}118 \pm 37 * \\
(\text { Bromage }=0)\end{array}$ & $218^{a}(56)$ & 0 & & 0 & \\
\hline $\begin{array}{l}163 \pm 42 \text { (complete } \\
\text { regression) }\end{array}$ & $\begin{array}{l}\text { I57 (4I) } \\
(\text { Bromage=0) }\end{array}$ & 277 (85) & 0 & & 0 & \\
\hline $\begin{array}{l}56(20-153) \text { (for } \\
\text { 2-dermatome } \\
\text { regression) }\end{array}$ & $\begin{array}{l}184 \pm 46 \\
(\text { Bromage }=0)\end{array}$ & $227 \pm 45$ & 3 & $\begin{array}{l}\text { A maximum } \\
\text { of } 500 \mathrm{~mL} \\
\text { crystalloids }\end{array}$ & 0 & $\begin{array}{l}\text { Articaina showed a faster full motor } \\
\text { function recovery and a shorter time for } \\
\text { spontaneous micturition }\end{array}$ \\
\hline $\begin{array}{l}61 \text { (24-104) (for } \\
\text { 2-dermatome } \\
\text { regression) }\end{array}$ & $\begin{array}{l}140 \pm 33^{*} \\
(\text { Bromage }=0)\end{array}$ & $184 \pm 39^{a}$ & 1 & & 1 & \\
\hline $\begin{array}{l}240 \pm 90 * \\
\text { (SI regression) }\end{array}$ & $\begin{array}{l}135 \pm 90 * \\
(\text { Bromage }=0)\end{array}$ & $306 \pm 56^{a}$ & 0 & $\begin{array}{l}\mathrm{I}, 000 \mathrm{~mL} \\
\text { crystalloids }\end{array}$ & 0 & $\begin{array}{l}\text { Hyperbaric } 2 \% \text { prilocaine is superior } \\
\text { to hyperbaric } 0.5 \text { bupivacaine due to a }\end{array}$ \\
\hline $\begin{array}{l}360 \pm 60 \\
(\mathrm{SI} \text { regression) }\end{array}$ & $\begin{array}{l}210 \pm 90 \\
(\text { Bromage }=0)\end{array}$ & $405 \pm 125$ & 2 & before SA & 0 & $\begin{array}{l}\text { shorter effect profile with equivalent } \\
\text { quality of block }\end{array}$ \\
\hline
\end{tabular}


Table I (Continued)

\begin{tabular}{|c|c|c|c|c|c|c|c|}
\hline Author & $\begin{array}{l}\text { Jadad } \\
\text { Scale }\end{array}$ & Drugs & Additives & Pts & Setting & $\begin{array}{l}\text { Sensory block } \\
\text { onset (min) }\end{array}$ & $\begin{array}{l}\text { Motor block } \\
\text { onset (min) }\end{array}$ \\
\hline \multirow[t]{2}{*}{$\begin{array}{l}\text { De Weert } \\
\text { et } \mathrm{al}^{50}\end{array}$} & 3 & $80 \mathrm{mg} 2 \% \mathrm{P}$ prilocaine & None & 35 & $\begin{array}{l}\text { Surgical procedures } \\
\text { lasting }<60 \mathrm{~min}\end{array}$ & NR & NR \\
\hline & & $80 \mathrm{mg} 2 \% \mathrm{P}$ lidocaine & None & 35 & & NR & NR \\
\hline \multirow[t]{2}{*}{$\begin{array}{l}\text { Ostgaard } \\
\text { et al }\left.\right|^{51}\end{array}$} & 5 & $80 \mathrm{mg} 2 \% \mathrm{P}$ prilocaine & None & 50 & $\begin{array}{l}\text { Urologic surgical } \\
\text { procedures lasting }\end{array}$ & $\begin{array}{l}\text { 13.4 } \pm 4 \text { (higher } \\
\text { dermatome) }\end{array}$ & NR \\
\hline & & $80 \mathrm{mg} 2 \% \mathrm{P}$ lidocaine & None & 50 & $<60 \min$ & $\begin{array}{l}14.5 \pm 6 \text { (higher } \\
\text { dermatome) }\end{array}$ & NR \\
\hline \multirow[t]{5}{*}{$\begin{array}{l}\text { Hampl } \\
\text { et } \mathrm{al}^{47}\end{array}$} & 4 & $\begin{array}{l}50 \mathrm{mg} 2 \% \mathrm{H}(7.5 \% \\
\text { glucose) prilocaine }\end{array}$ & None & 30 & $\begin{array}{l}\text { Gynecologic short } \\
\text { surgical procedures }\end{array}$ & NR & $4(I-4)$ \\
\hline & & $50 \mathrm{mg} 2 \% \mathrm{H}(7.5 \%$ & None & 30 & & NR & $4(2-4)$ \\
\hline & & glucose) lidocaine & & & & & \\
\hline & & $12.5 \mathrm{mg} 0.5 \% \mathrm{H}(7.5 \%$ & None & 30 & & NR & $4(1-4)$ \\
\hline & & glucose) bupivacaine & & & & & \\
\hline
\end{tabular}

Note: aSignificant difference. *Statistically significant.

Abbreviations: $\mathrm{H}$, hyperbaric; NR, not reported; P, plain; POUR postoperative urinary retention; Pts, patients; SA, spinal anesthesia; TNS, transient neurologic symptoms.

(178 min with prilocaine vs $195 \mathrm{~min}$ with mepivacaine) as well as faster discharge time (192 vs $220 \mathrm{~min}$ ). In the prilocaine group, the time to first analgesic administration was 173 min. $^{28}$

In day-case perianal surgery, Kaban compared $30 \mathrm{mg}$ $2 \%$ hyperbaric prilocaine with fentanyl $20 \mu \mathrm{g}$ versus $7.5 \mathrm{mg}$ of $0.5 \%$ hyperbaric bupivacaine with fentanyl $20 \mu \mathrm{g} .{ }^{29} \mathrm{Time}$ to unassisted ambulation, time to sensory block resolution, and time to home discharge were significantly shorter in the prilocaine group than in the bupivacaine group. One patient in each group had urinary retention. Compared to the study by Gebhardt, prilocaine $30 \mathrm{mg}$ showed a shorter time to urinary voiding ( $152 \pm 104$ vs $211 \pm 33)$ and to discharge $(155 \pm 100$ vs $229 \pm 32)$. As the author noticed, in the study by Gebhardt, the patients waited in the sitting position for 10 min after the spinal injection, whereas in the study by Kaban the patients remained in sitting position for only 2 min after the injection. As a consequence, in the first case, the anesthetic spread a median of 5 dermatomes from S5 upwards (max to L4 dermatome), whereas the anesthetic spread up to T9 dermatome in the second case. As the duration of spinal nerve blockade is inversely related to the intrathecal spread of the same anesthetic dosage,${ }^{21}$ in the study by Kaban spinal recovery resulted faster.

The author also highlighted that in the prilocaine group, despite the shorter sensory block recovery $(133 \pm 41 \mathrm{~min}$ to $\mathrm{S} 3$ dermatome resolution), the time to first analgesic intake was almost delayed (192 $\mathrm{min}$ ), promoting fentanyl $20 \mu \mathrm{g}$ as a suitable adjuvant with the aim to increase the quality of the sensory block without prolonging motor block and time to micturition.

\section{Arthroscopy knee surgery}

In arthroscopy knee surgery, Guntz et al performed a dose finding study using $2 \%$ hyperbaric prilocaine. ${ }^{30}$ With the up-and-down sequential allocation technique, Guntz estimated the effective dose 90 of $2 \%$ hyperbaric prilocaine to be $38.5 \mathrm{mg}$ for patients undergoing knee arthroscopy under spinal anesthesia. The author suggested $40 \mathrm{mg}$ as the dose required to provide an adequate sensory (T12) and motor block (Bromage=3) in 92\% of the patients, 15 min after spinal injection. Moreover, in the same study $40 \mathrm{mg}$ of $2 \%$ hyperbaricc prilocaine showed hemodynamic stability, motor block regression in less than $90 \mathrm{~min}(87 \mathrm{~min})$, and spontaneous voiding in all patients enrolled leading to a time of eligibility for home discharge of $205 \mathrm{~min}$.

In day-case knee arthroscopy, $40 \mathrm{mg}$ was the dose chosen by Ambrosoli et al to compare intrathecal blockade with $2 \%$ hyperbaric prilocaine versus ultrasound-guided femoralsciatic nerve blockade with mepivacaine $2 \% .{ }^{31}$ Sensory and motor blockade recovered sooner after prilocaine spinal anesthesia. Time to home readiness was faster after intrathecal blockade than after peripheral nerve blockade, while time to micturition was not different between the two techniques (225 min after intrathecal anesthesia vs $220 \mathrm{~min}$ after periph- 


\begin{tabular}{|c|c|c|c|c|c|c|}
\hline $\begin{array}{l}\text { Sensory block } \\
\text { resolution ( } \mathrm{min})\end{array}$ & $\begin{array}{l}\text { Motor block } \\
\text { resolution (min) }\end{array}$ & $\begin{array}{l}\text { Time to } \\
\text { micturition (min) }\end{array}$ & POUR & $\begin{array}{l}\text { Fluid } \\
\text { management }\end{array}$ & TNS & Main results \\
\hline $\begin{array}{l}127 \pm 59 \text { (for } \\
\text { 2-dermatome } \\
\text { regression) }\end{array}$ & $\begin{array}{l}166 \pm 45 \\
(\text { Bromage }=0)\end{array}$ & NR & NR & $\begin{array}{l}500 \mathrm{~mL} 0.45 \% \\
\text { saline/3.3\% } \\
\text { glucose solution }\end{array}$ & 0 & $\begin{array}{l}\text { Prilocaine results in a lower incidence } \\
\text { of transient neurological symptoms than } \\
\text { lidocaine intrathecally and therefore }\end{array}$ \\
\hline $\begin{array}{l}105 \pm 39 \text { (for } \\
\text { 2-dermatome } \\
\text { regression) }\end{array}$ & $\begin{array}{l}130 \pm 30 \\
(\text { Bromage }=0)\end{array}$ & NR & NR & before SA & 7 & $\begin{array}{l}\text { it is more suitable for short surgical } \\
\text { procedures }\end{array}$ \\
\hline $\begin{array}{l}221 \pm 49 \\
(\mathrm{SI} \text { regression })\end{array}$ & $\begin{array}{l}197 \pm 42 \\
(\text { Bromage }=0)\end{array}$ & NR & NR & $\begin{array}{l}500 \mathrm{~mL} \\
\text { crystalloids }\end{array}$ & 2 & $\begin{array}{l}\text { Isobaric prilocaine has a longer duration } \\
\text { of action than an equal dose of lidocaine }\end{array}$ \\
\hline $\begin{array}{l}I 8 I \pm 48 \\
\text { (SI regression) }\end{array}$ & $\begin{array}{l}\text { I53 } 546 \\
(\text { Bromage }=0)\end{array}$ & NR & NR & before SA & 7 & $\begin{array}{l}\text { and may be an alternative drug for spinal } \\
\text { anesthesia of intermediate or short } \\
\text { duration. TNS occurred also after the } \\
\text { isobaric prilocaine spinal anesthesia; there } \\
\text { may be an indication of a lower frequency }\end{array}$ \\
\hline $\begin{array}{l}128 \pm 38 \\
\text { (SI regression) }\end{array}$ & $\begin{array}{l}165 \pm 37 \\
(\text { Bromage }=0)\end{array}$ & $253 \pm 55$ & NR & NR & 9 & $\begin{array}{l}\text { Prilocaine was associated with a } \\
\text { significantly lower incidence of TNS }\end{array}$ \\
\hline $\begin{array}{l}127 \pm 33 \\
\text { (S2 regression) }\end{array}$ & $\begin{array}{l}155 \pm 40 \\
\text { (Bromage }=0)\end{array}$ & $238 \pm 57$ & NR & & I & $\begin{array}{l}\text { compared with lidocaine. The duration } \\
\text { of action was comparable to that of }\end{array}$ \\
\hline $\begin{array}{l}172 \pm 42 \\
\text { (S2 regression) }\end{array}$ & $\begin{array}{l}200 \pm 48 \\
(\text { Bromage }=0)\end{array}$ & $299 \pm 85$ & NR & & 0 & $\begin{array}{l}\text { lidocaine. Prilocaine might be appropriate } \\
\text { to use in place of lidocaine for spinal } \\
\text { anesthesia }\end{array}$ \\
\hline
\end{tabular}

eral nerve blockade). The study confirmed prilocaine $40 \mathrm{mg}$ to be adequate for knee arthroscopy in $96 \%$ of the patients.

In the same type of ambulatory surgery, Aguirre et al compared $60 \mathrm{mg}$ of $2 \%$ hyperbaric prilocaine with $12 \mathrm{mg}$ of $0.4 \%$ plain ropivacaine. ${ }^{32}$ The offset of motor block was faster after intrathecal administration of prilocaine (180 vs $240 \mathrm{~min}$ ). Nevertheless, $60 \mathrm{mg}$ of prilocaine did not show any difference regarding first spontaneous voiding and discharge times between the two groups (330 min with prilocaine vs 335 min with ropivacaine). According to Manassero et al, this result was mainly influenced by the high selected dose of prilocaine $(60 \mathrm{mg}$ ) for knee arthroscopy and, moreover, not equipotent to ropivacaine $12 \mathrm{mg} .{ }^{33,34}$

Hendriks compared $50 \mathrm{mg}$ of $2 \%$ plain prilocaine to 50 $\mathrm{mg}$ of $2 \%$ plain articaine in day-case knee arthroscopy. ${ }^{35}$ Recovery of motor function ( $140 \pm 33$ vs $184 \pm 46 \mathrm{~min}$ ) and time to spontaneous voiding ( $184 \pm 33$ vs $227 \pm 45 \mathrm{~min}$ ) were significantly shorter with articaine.

Using the plain prilocaine formulation, Black compared a remarkably low dose of $20 \mathrm{mg}$ with fentanyl $20 \mu \mathrm{g}$ versus $7.5 \mathrm{mg}$ plain bupivacaine with fentanyl $20 \mu \mathrm{g}$ in knee arthroscopies with median time elapsed from intrathecal drug administration to arrival in the recovery area of 35 (20-55) min. Time to micturition, motor, and sensory block regression was significantly shorter in prilocaine than in bupivacaine group. ${ }^{36}$ A total of six patients (12\%) complained of pruritus.

\section{Urologic procedures}

In geriatric patients undergoing day-case transurethral resection of the prostate (TURP) surgery, Akcaboy matched intrathecal administration of a conventional dose of $50 \mathrm{mg}$ $2 \%$ hyperbaric prilocaine plus fentanyl $25 \mu \mathrm{g}$ and a low dose of $4 \mathrm{mg} 0.5 \%$ hyperbaric bupivacaine plus fentanyl $25 \mu \mathrm{g} .{ }^{37}$ Dermatome T10 was the desired level of analgesia. Despite the very low dose of bupivacaine, none of the patients in either group manifested block failure or pain during the entire procedure which lasted a mean of $60 \mathrm{~min}$. Spinal anesthesia with bupivacaine recovered sooner, while adverse events such hypotension and bradycardia were observed more frequently in the prilocaine group. A total of six patients $(12 \%)$ complained of pruritus.

The author concluded $4 \mathrm{mg}$ bupivacaine plus fentanyl $25 \mu \mathrm{g}$ are comparable to $50 \mathrm{mg}$ prilocaine plus fentanyl $25 \mu \mathrm{g}$ in TURP. Nevertheless, the author commented that the comparison might be affected by not equipotent dose selection.

\section{Prilocaine and postoperative urinary retention}

It is well known that, after spinal anesthesia, spontaneous voiding is the last function to recover after motor block resolution. It may not be expected until regression of sensory blockade reaches S3 dermatome level. Moreover, the contents of the bladder can exceed the cystometric capacity before the reappearing of its normal function, leading to an acute 
postoperative urinary retention (POUR).$^{38}$ Compared to an equipotent dose of long-acting spinal anesthetic, prilocaine showed a more rapid return of bladder function associated with lower incidence of POUR, as expected with shorteracting drugs. ${ }^{23,36}$

In 25 day-case perianal surgeries, Kaban showed one case of POUR after intrathecal $30 \mathrm{mg}$ of $2 \%$ hyperbaric prilocaine with fentanyl $20 \mu \mathrm{g} .{ }^{29}$ Using 40 or $50 \mathrm{mg} 2 \%$ hyperbaric prilocaine, mean time to first micturition ranged from 195 to 249 min with no cases of POUR in 160 patients. ${ }^{22,26,31}$

With $60 \mathrm{mg}$ of hyperbaric 2\% prilocaine, Aguirre, Camponovo and Ratsh showed no case of POUR in 144 lower limb ambulatory surgeries, reporting mean times to first micturition ranging between 218 and 306 min..$^{22,23,32}$ Nevertheless, after a properly designed observational study, using the same dosage of intrathecal $2 \%$ hyperbaric prilocaine, Kreutziger et al showed a high rate of urinary retention (23\%) in 86 relatively low-risk patients (mean age 46 years without urogenital pathologies, lower limb minor orthopedic procedures). As per the study protocol, catheterization was planned when bladder volume exceeded $600 \mathrm{~mL}$ together with the inability to micturate. ${ }^{39}$ To avoid a dangerous bladder over-distension, the author outlined the importance to use restrict criteria for catheterization based not on a clinical judgment but on ultrasound estimation of the bladder volume. ${ }^{40}$ If spontaneous micturition should remain a criterion for discharge after day-case prilocaine, spinal anesthesia is still debated. Risk factors for postoperative urinary retention are well identified. ${ }^{41}$ Short-acting spinal anesthetics for low-risk patients and low-risk procedures, are associated with minimal risk of urinary retention, and the patient could be discharged home without the need to void before discharge. ${ }^{42}$ Patients at high risk of urinary retention should be managed with ultrasound bladder volume estimation, requiring voiding before discharge or catheterization if the bladder volume exceeded $600 \mathrm{~mL} .^{2}$ Any case, providing adequate but not excessive intravenous fluid perioperatively may overall, minimize the risk for POUR (Table 1). ${ }^{42}$

\section{Prilocaine and TNS}

The symptoms of TNS can appear in few hours postoperatively or within the first $24 \mathrm{~h}$, even after recovery from an uneventful spinal block. These symptoms consist of pain originating in the gluteal region and radiating to lower extremities. ${ }^{43-46}$

Prilocaine was extensively examined especially for the incidence of TNS after subarachnoid use. In a prospective, double-blinded study, Hampl et al compared prilocaine to lidocaine and bupivacaine for the relative risk of TNS. ${ }^{47}$
Spinal anesthesia was induced with $2.5 \mathrm{~mL} 2 \%$ lidocaine in $7.5 \%$ glucose, $2 \%$ prilocaine in $7.5 \%$ glucose, or $0.5 \%$ bupivacaine in $7.5 \%$ glucose. Symptoms of TNS were observed for 9 patients (30\%) receiving lidocaine, 1 patient receiving prilocaine (3\%), and none receiving bupivacaine. The difference in the incidence of TNS between lidocaine and prilocaine was statistically significant.

Two hundred patients were randomly treated by MartìnezBourio et al with $5 \%$ hyperbaric prilocaine or $5 \%$ hyperbaric lidocaine. The dose for spinal anesthesia was calculated in relation to the type of procedure and patient height (mean dose: $68.6 \mathrm{mg}$ for prilocaine and $67.7 \mathrm{mg}$ for lidocaine). TNS occurred within $12-24 \mathrm{~h}$ in 1 patient $(1 \%)$ in the prilocaine group and 4 patients $(4.2 \%)$ in the lidocaine group and disappeared in both groups within 48-72 h. The difference was not significant, also due to the low incidence of the symptoms in this population. ${ }^{48}$

A survey on about 5,000 spinal anesthesia performed with $1 \mathrm{mg} / \mathrm{kg} 2 \%$ prilocaine did not report any case of TNS. ${ }^{49}$

Another study compared $80 \mathrm{mg}$ of $2 \%$ prilocaine plain solutions versus $2 \%$ lidocaine plain solutions for spinal anesthesia. ${ }^{50}$ Seven patients (20\%) treated with lidocaine had TNS within $24 \mathrm{~h}$ after surgery and symptoms disappeared within 4 days. In contrast, no patient in the prilocaine group reported TNS and the difference was statistically significant.

With the same doses of plain prilocaine and lidocaine formulation ( $80 \mathrm{mg}, 2 \%$ solution), Østgaard et al performed a study on 100 patients scheduled for elective short urologic procedures. ${ }^{51}$ Nine patients fulfilled the criteria for TNS: 7 in the lidocaine group (14\%) and 2 in the prilocaine group (4\%). Symptoms resolved within 2-3 days, but the difference was not significant. Moreover, both studies showed prilocaine as a same duration of action than an equal dose of lidocaine resulting an alternative drug for spinal anesthesia of intermediate or short duration.

The risk of TNS after spinal anesthesia with local anesthetics was evaluated by a systematic review of randomized controlled trials. ${ }^{52}$ A total of 29 studies (2813 patients) was evaluated in the analysis: the incidence of TNS was $16.9 \%$ after lidocaine, $19.1 \%$ after mepivacaine, $1.1 \%$ after bupivacaine, and $1.7 \%$ after prilocaine. The relative risk for TNS resulted to be 6.7- and 5.5-fold higher for lidocaine than bupivacaine and prilocaine, respectively. Furthermore, data showed that baricity and concentration of the local anesthetic have no significant influence on the occurrence of TNS.

A similar systematic analysis was performed to compare the frequency of TNS after spinal anesthesia with lidocaine versus other local anesthetics in adult surgical patients. ${ }^{53,54}$ Fourteen 
studies enrolling a total of 1,349 patients were examined: 117 patients developed TNS. It was evinced that all these drugs can cause TNS. However, the relative risk for developing TNS after spinal anesthesia with lidocaine was 4.35 , as compared to other local anesthetics (bupivacaine, prilocaine, procaine, levobupivacaine, and ropivacaine). Finally, an updated analysis was conducted in 2009, confirming the lower risk of TNS related to spinal prilocaine compared to lidocaine..$^{55}$

\section{Conclusion}

Due to its predictable intermediate duration of action and the low incidence of TNS (Evidence $\mathrm{Ia}^{56}$ ), spinal $2 \%$ hyperbaric prilocaine can be successfully used to provide anesthesia for a variety of day-case procedures, resulting an alternative drug to lidocaine and mepivacaine for spinal anesthesia of intermediate or short duration. ${ }^{49-55}$

Compared to an equipotent dose of long-acting spinal anesthetic, prilocaine showed a more rapid recovery and return of bladder function, associated with lower incidence of POUR (Evidence Ib). ${ }^{23,29,36}$ Future trials are advocated to establish possible differences between prilocaine over other local anesthetics with similar favorable and safety profile, such as articaina and chloroprocaine.

The dose of prilocaine has to be related to the type of surgery, patient's characteristics, and local discharge criteria. Literature suggests a dose ranging between 40 and $60 \mathrm{mg}$ for lower extremities and lower abdominal procedures lasting up to $90 \mathrm{~min}$ and $10 \mathrm{mg}$ of $2 \%$ hyperbaric prilocaine for minor perianal surgery. Readiness for discharge occurs in about $4 \mathrm{~h}$ from spinal administration. In comparison with plain solutions, hyperbaricity remarkably accelerates the onset and offset times of intrathecal anesthesia.

Although the combination of prilocaine and fentanyl (20-25 $\mu \mathrm{g}$ ) has been tested with the aim to improve the quality and extend the duration of the spinal block, pruritus and urinary retention are possible side effects which can delay home discharge. ${ }^{29,36,37}$

\section{Limitations}

Limitations of the present review are represented by the relatively small number of RCTs evaluating $2 \%$ hyperbaric prilocaine compared to any other local anesthetic, together with the heterogeneity of the doses used and the outcomes investigated, which does not permit a rigorous evidence-based evaluation of its advantages in outpatient spinal anesthesia.

\section{Disclosure}

The authors report no conflicts of interest in this work.

\section{References}

1. Förster JG. Short-acting spinal anesthesia in the ambulatory setting. Curr Opin Anaesthesiol. 2014;27(6):597-604.

2. Schneider M, Ettlin T, Kaufmann M, et al. Transient neurologic toxicity after hyperbaric subarachnoid anesthesia with 5\% lidocaine. Anesth Analg. 1993;76(5):1154-1157.

3. Tarkkila P, HuhtalaJ, Tuominen M. Transient radicular irritation after spinal anesthesia with hyperbaric 5\% lignocaine. Br J Anaesth. 1995;74(3):328-329.

4. Hiller A, Rosenberg PH. Transient neurological symptoms after spinal anaesthesia with $4 \%$ mepivacaine and $0.5 \%$ bupivacaine. Br JAnaesth. 1997;79(3):301-305.

5. Freedman JM, Li DK, Drasner K, Jaskela MC, Larsen B, Wi S. Transient neurologic symptoms after spinal anesthesia: an epidemiologic study of 1,863 patients. Anesthesiology. 1998;89(3):633-641.

6. Hodgson PS, Liu SS, Batra MS, Gras TW, Pollock JE, Neal JM. Procaine compared with lidocaine for incidence of transient neurologic symptoms. Reg Anesth Pain Med. 2000;25(3):218-222.

7. Liu SS, Ware PD, Allen HW, Neal JM, Pollock JE. Dose-response characteristics of spinal bupivacaine in volunteers. Clinical implications for ambulatory anesthesia. Anesthesiology. 1996;89(6): 729-736.

8. Nair GS, Abrishami A, Lermitte J, Chung F. Systematic review of spinal anaesthesia using bupivacaine for ambulatory knee arthroscopy. $\mathrm{Br} \mathrm{J}$ Anaesth. 2009;102(3):307-315.

9. Jadad AR, Moore RA, Carroll D, et al. Assessing the quality of reports of randomized clinical trials: is blinding necessary? Control Clin Trials. 1996;17(1):1-12.

10. Tucker GT, Mather LE. Clinical Pharmacokinetics of local anaesthetics. Clin Pharmacokinet. 1979;4(4):241-278.

11. Hardman JD, Limbird LE, Molinoff RW, Ruddon AG. In: Goodman LS, Gilman A, editors. The Pharmacological Basis of Therapeutics. New York, NY: McGraw-Hill; 1996:338.

12. Vasters FG, Eberhart LH, Koch T, Kranke P, Wulf H, Morin AM. Risk factors for prilocaine-induced methaemoglobinaemia following peripheral regional anaesthesia. Eur J Anaesthesiol. 2006;23(9): 760-765.

13. Crankshaw TP. Citanest (Prilocaine) in spinal analgesia. Acta Anaesth Scand. 1965;9(s16):287-290.

14. Hillmann KM. Spinal prilocaine. Anaesthesia. 1978;33(1):68-69.

15. Robertson DH. Spinal prilocaine. Anaesthesia. 1978;33(7):647-648.

16. Hocking G, Wildsmith JA. Intrathecal drug spread. Br J Anaesth. 2004;93(4):568-578.

17. McLeod GA. Density of spinal anaesthetic solutions of bupivacaine, levobupivacaine and ropivacaine with and without dextrose. $\mathrm{Br} \mathrm{J}$ Anaesth. 2004;92(4):547-551.

18. Bachmann M, Pere P, Kairaluoma P, Rosenberg PH, Kallio H. Comparison of hyperbaric and plain articaine in spinal anaesthesia for open inguinal hernia repair. Br J Anaesth. 2008;101(6):848-854.

19. Sen H, Purtuloglu T, Sizlan A, et al. Comparison of intrathecal hyperbaric and isobaric levobupivacaine in urological surgery. Minerva Anestesiol. 2010;76(1):24-28.

20. Fettes P, Hocking G, Peterson M, Luck JF, Wildsmith JA. Comparison of plain and hyperbaric solutions of ropivacaine for spinal anaesthesia. Br J Anaesth. 2005;94(1):107-111.

21. Kooger Infante NE, Van Gessel E, Forster A, Gamulin Z. Extent of hyperbaric spinal anesthesia influences the duration of spinal block. Anesthesiology. 2000;92(5):1319-1323.

22. Camponovo C, Fanelli A, Ghisi D, Cristina D, Fanelli G. A prospective, prospective, double-blinded, randomized, clinical trial comparing the efficacy of $40 \mathrm{mg}$ and $60 \mathrm{mg}$ hyperbaric 2prilocaine versus $60 \mathrm{mg}$ plain prilocaine for intathecal anesthesia in ambulatory surgery. Anesth Analg. 2010;111(2):568-572.

23. Rätsch G, Niebergall H, Hauenstein L, Reber A. Spinal anaesthesia in day-case surgery. Optimisation of procedures. Anaesthesist. 2007; 56(4):322-327. 
24. Enk D, Prien T, Van Aken H, Mertes N, Meyer J, Brüssel T. Success rate of unilateral spinal anesthesia is dependent on injection flow. Reg Anesth Pain Med. 2001;26(5):420-427.

25. Casati A, Fanelli G, Aldegheri G, et al. Frequency of hypotension during conventional or asymmetric hyperbaric spinal block. Reg Anesth Pain Med. 1999;24(3):214-219.

26. Manassero A, Bossolasco M, Ugues S, Bailo C, Liarou C, Coletta G. Comparison of unilateral and bilateral spinal anesthesia with $2 \%$ hyperbaric prilocaine in day-case inguinal herniorraphy: a randomized controlled trial. Minerva Anestesiol. 2014;80(6):685-691.

27. Gebhardt V, Herold A, Weiss C, Samakas A, Schmittner MD. Dosage finding for low-dose spinal anaesthesia using hyperbaric prilocaine in patients undergoing perianal outpatient surgery. Acta Anaesthesiol Scand. 2013;57(2):249-256.

28. Gebhardt V, Beilstein B, Herold A, et al. Spinal hyperbaric prilocaine versus. mepivacaine in perianal outpatient surgery. Central Eur J Med. 2014;9(6):754-761.

29. Kaban OG, Yazicioglu D, Akkaya T, Sayin MM, Seker D, Gumus H. Spinal anaesthesia with hyperbaric prilocaine in day-case perianal surgery: randomised controlled trial. Scientific World Journal. 2014;2014:608372.

30. Guntz E, Latrech B, Tsiberidis C, Gouwy J, Kapessidou Y. ED 50 and ED 90 of intrathecal hyperbaric $2 \%$ prilocaine in ambulatory knee arthroscopy. Can J Anesth. 2014;61(9):801-807.

31. Ambrosoli AL, Chiaranda M, Fedele LL, Gemma M, Cedrati V, Cappelleri GA. Randomised controlled trial of intrathecal blockade versus peripheral nerve blockade for day-case knee arthroscopy. Anaesthesia. 2016;71(3):280-284.

32. Aguirre J, Borgeat A, Bühler P, Mrdjen J, Hardmeier B, Bonvini JM. Intrathecal hyperbaric $2 \%$ prilocaine versus $0.4 \%$ plain ropivacaine for same-day arthroscopic knee surgery: a prospective randomized, double-blind controlled study. Can J Anesth. 2015;62(10):1055-1062.

33. Manassero A, Meconi T, Fanelli A. Is $60 \mathrm{mg}$ a suitable dosage for sameday spinal prilocaine? Can J Anesth. 2016;63(4):495-496.

34. Guntz E, Kapessidou Y. Spinal prilocaine for same-day surgery: the importance of equipotent doses. Can J Anesth. 2016;63(8):985-986.

35. Hendriks MP, De Weert CJM, Snoeck MMJ, Hu HP, Pluim MAL, Gielen MJM. Plain articaine or prilocaine for spinal anaesthesia in daycase knee arthroscopy: a double-blind randomized trial. Br J Anaesth. 2009;102(2):259-263.

36. Black AS, Newcombe GN, Plummer JL, McLeod DH, Martin DK. Spinal anaesthesia for ambulatory arthroscopic surgery of the knee: a comparison of low-dose prilocaine and fentanyl with bupivacaine and fentanyl. Br J Anaesth. 2011;106(2):183-188.

37. Akcaboy ZN, Akcaboy ET, Mutlu NM, Serger N, Aksu C, Gogus N. Spinal anesthesia with low-dose bupivacaine-fentanyl combination: a good alternative for day case transurethral resection of prostate surgery in geriatric patients. Rev Bras Anestesiol. 2012;62(6):753-761.

38. Kamphuis ET, Ionescu TI, Kuipers PW, de Gier J, van Venrooij GE, Boon TA. Recovery of storage and emptying functions of the urinary bladder after spinal anesthesia with lidocaine and with bupivacaine in men. Anesthesiology. 1998;88(2):310-316.

39. Kreutziger J, Frankenberger B, Luger TJ, Richard S, Zbinden S. Urinary retention after spinal anaesthesia with hyperbaric prilocaine $2 \%$ in an ambulatory setting. Br J Anaesth. 2010;104(5):582-586.
40. Pavlin DJ, Pavlin EG, Gunn HC, Taraday JK, Koerschgen ME. Voiding in patients managed with or without ultrasound monitoring of bladder volume after outpatient surgery. Anesth Analg. 1999;89(1):90-97.

41. Awad IT, Chung F. Factors affecting recovery and discharge following ambulatory surgery. Can J Anesth. 2006;53(9):858-872.

42. Mulroy MF, Salinas FV, Larkin KL, Polissar NL. Ambulatory surgery patients may be discharged before voiding after short-acting spinal and epidural anesthesia. Anesthesiology. 2002;97(2)315-319.

43. Hampl KF, Schneider MC, Ummenhofer W, Drewe J. Transient neurologic symptoms after spinal anesthesia. Anesth Analg. 1995;81(6): $1148-1153$

44. Hampl KF, Schneider MC, Pargger H, Gut J, Drewe J, Drasner K. A similar incidence of transient neurologic symptoms after spinal anesthesia with 2\% and 5\% lidocaine. Anesth Analg. 1996;83(5):1051-1054.

45. Pollok JE. Transient neurologic symptoms: etiology, risk factors, and management. Reg Anesth Pain Med. 2002;27(6):581-586.

46. Pollock JE. Neurotoxicity of intrathecal local anaesthetics and transient neurological symptoms. Best Pract Res Clin Anaesthesiol. 2003; 17(3)471-484.

47. Hampl KF, Heinzmann-Wiedmer S, Luginbuehl I, et al. Transient neurologic symptoms after spinal anestesia: a lower incidence with prilocaine and bupivacaine than with lidocaine. Anesthesiology. 1988;88(3):629-633.

48. Martìnez-Bourio R, Arzuaga M, Quintana JM, et al. Incidence of transient neurologic symptoms after hyperbaric subarachnoid anesthesia with 5\% lidocaine and 5\% prilocaine. Anesthesiology. 1998;88(3):624-628.

49. König W, Ruzicic D. Absence of transient radicular irritation after 5000 spinal anaesthetics with prilocaine. Anaesthesia. 1997;52(2):182-183.

50. De Weert K, Traskel M, Gielen M, Slappendel R, Weber E, Dirksen R. The incidence of transient neurological symptoms after spinal anaesthesia with lidocaine compared to prilocaine. Anaesthesia. 2000;55(10):1020-1024.

51. Østgaard G, Hallaråker O, Ulveseth OK, Flaatten H. A randomised study of lidocaine and prilocaine for spinal anaesthesia. Acta Anaesthesiol Scand. 2000;44(4):436-440.

52. Eberhart LH, Morin AM, Kranke P, Geldner G, Wulf H. Transient neurologic symptoms after spinal anesthesia. A quantitative systematic review (meta-analysis) of randomized controlled studies. Anaesthesist. 2002;51:539-546.

53. Zaric D, Pace NL, Christiansen C, Punjasawadwong Y. Transient neurological symptoms (TNS) following spinal anaesthesia with lidocaine versus other local anaesthetics. Cochrane Database Syst Rev. 2003;(2):CD003006.

54. Zaric D, Christiansen C, Pace NL, Punjasawadwong Y. Transient neurological symptoms (TNS) following spinal anaesthesia with lidocaine versus other local anaesthetics: a systematic review of randomized, controlled trials. Anaesth Analg. 2005;100(6):1811-1816.

55. Zaric D, Pace NL. Transient neurologic symptoms (TNS) following spinal anaesthesia with lidocaine versus other local anaesthetics. Cochrane Database Syst Rev. 2009;15(2):CD003006.

56. cebm.net [homepage on the Internet]. University of Oxford: The Centre for Evidence-Based Medicine; November 1998 [Updated by Jeremy Howick March 2009]. Available from: http://www.cebm.net/ oxford-centre-evidence-based-medicine-levels-evidence-march-2009/. Accessed February 26, 2017.
Local and Regional Anesthesia

\section{Publish your work in this journal}

Local and Regional Anesthesia is an international, peer-reviewed, open access journal publishing on the development, pharmacology, delivery and targeting and clinical use of local and regional anesthetics and analgesics. The journal is included in PubMed, and welcomes submitted papers covering original research, basic science, clinical studies,

\section{Dovepress}

reviews and evaluations, guidelines, expert opinion and commentary, case reports and extended reports. The manuscript management system is completely online and includes a very quick and fair peer-review system, which is all easy to use. Visit http://www.dovepress.com/ testimonials.php to read real quotes from published authors. 